\title{
AirSEM: Electron Microscopy in Air, without a Specimen Chamber
}

\author{
Kayla Nguyen ${ }^{1}$, Megan Holtz ${ }^{2}$ and David Muller ${ }^{2}$ \\ ${ }^{1 .}$ Department of Chemistry and Chemical Biology, Cornell University, Ithaca, NY, USA. \\ ${ }^{2}$ School of Applied and Engineering Physics, Cornell University, Ithaca, NY, USA.
}

The development of environmental scanning electron microscopes (ESEMs) has allowed the study of specimens in a gaseous environment. The ESEM typically operates at below atmospheric pressure due to the long gas path length and need for differential pumping apertures. A new generation of Atmospheric scanning electron microscopes (ASEMs) allow samples to be imaged in liquid or at atmospheric pressure through an electrontransparent window that separates the column of the microscope from the sample $[1,2]$. One approach to dealing with the short required working distance has been to image directly into the liquid with an inverted SEM column below a silicon nitride window held by a petri dish [1]. Here, we explore an alternative design for a general-purpose fieldemission AirSEM from b-Nano [2]. This is an upright geometry where the sample is mechanically positioned 50-200 microns below electron-transparent window after computer-controlled alignment with an optical microscope (Fig. 1a). This decouples the sample from the window, allowing for its reuse and enables multiple signal collection such as gas detection, in addition to the backscattered electron detector (BSE) [2,3].

The highest resolution images are obtained from interactions with the unscattered beam. Monte Carlo simulations [4] (Fig 1b) show the electron mean free path (mfp) changes from $75 \mu \mathrm{m}$ in air to $\sim 900 \mu \mathrm{m}$ in He. Therefore, in order to achieve high resolution, we placed our specimen $50 \mu \mathrm{m}$ away from the SiNx window and mix helium into the flow across this gap. With a standard $\mathrm{Au}$ on carbon resolution test sample, we were able to image Au nanoparticles smaller than $10 \mathrm{~nm}$ in BSE mode at 15 and $30 \mathrm{keV}$ (Fig 1c).

Due to the AirSEM's flexible design, we can work with in-situ cells and detectors with volumes several inches in each dimension without using vacuum feedthroughs, and samples can simply be placed on an optical microscope slide. The registration between the optical microscope and the electron beam is useful for correlative microscopy. Fig. 2 shows the comparison between optical and BSE images of a proton-exchange membrane fuel cell after 30,000 cycles of operation where there is coarsening and migration of the $\mathrm{Pt}_{3} \mathrm{Co}$ nanocatalysts, some of which have grown from their original $5 \mathrm{~nm}$ diameter to sizes of $10 \mathrm{~s}$ to $100 \mathrm{~s}$ of $\mathrm{nm}$. Fig 3 a shows the electrode of an aged Li-ion battery. Fig $3 \mathrm{~b}$ shows wet moss imaged on a glass side, illustrating the minimal preparation needed for wet biological samples. Fig $3 \mathrm{~b}$ shows a time sequence from the in-situ growth of $\mathrm{Pb}$ dendrites on the surface of a liquid. From these experiments, two notable features of the instrument are the rapid specimen turn-around time (a few minutes per sample) and the ready access for in-situ cells and detectors. [5]

\section{References:}

[1] M. Suga, C. Sato, et al., Ultramicroscopy 111 (2011) 1650-1658.

[2] b-Nano: http://www.b-nano.com/ 
[3] D. Stokes, Principles and Practice of Variable Pressure/Environmental Scanning Electron Microscopy. Wiley and Sons, West Sussex, (2008).

[4] D. Joy, Monte Carlo Modeling for Electron Microscopy and Microanalysis. Oxford University Press, NY, (1993). !!

[5] Work supported by the Energy Materials Center at Cornell, DOE EFRC BES (DESC0001086). Facility support from NSF MRSEC program (DMR 1120296) and the Kavli Institute at Cornell.
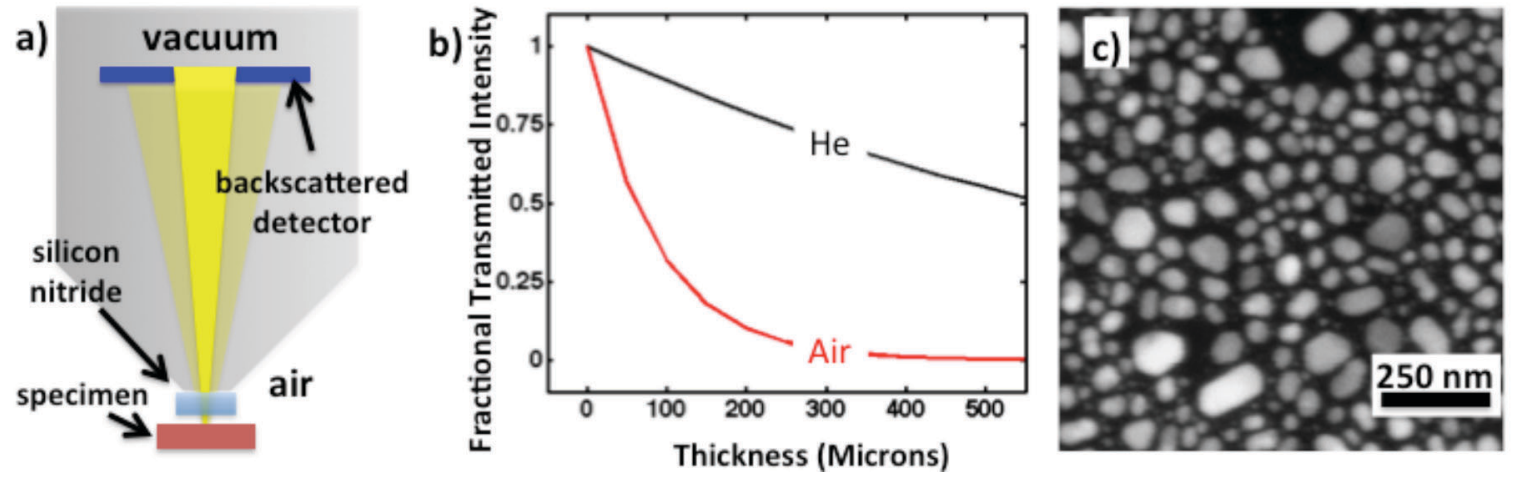

Figure 1. a) Schematic of the AirSEM. b) Fraction of the unscattered beam remaining as a function of distance through helium and air. c) Au nanoparticles as resolution testsmallest particles are $\sim 10 \mathrm{~nm}$ and are clearly resolved with smaller gaps also visible.
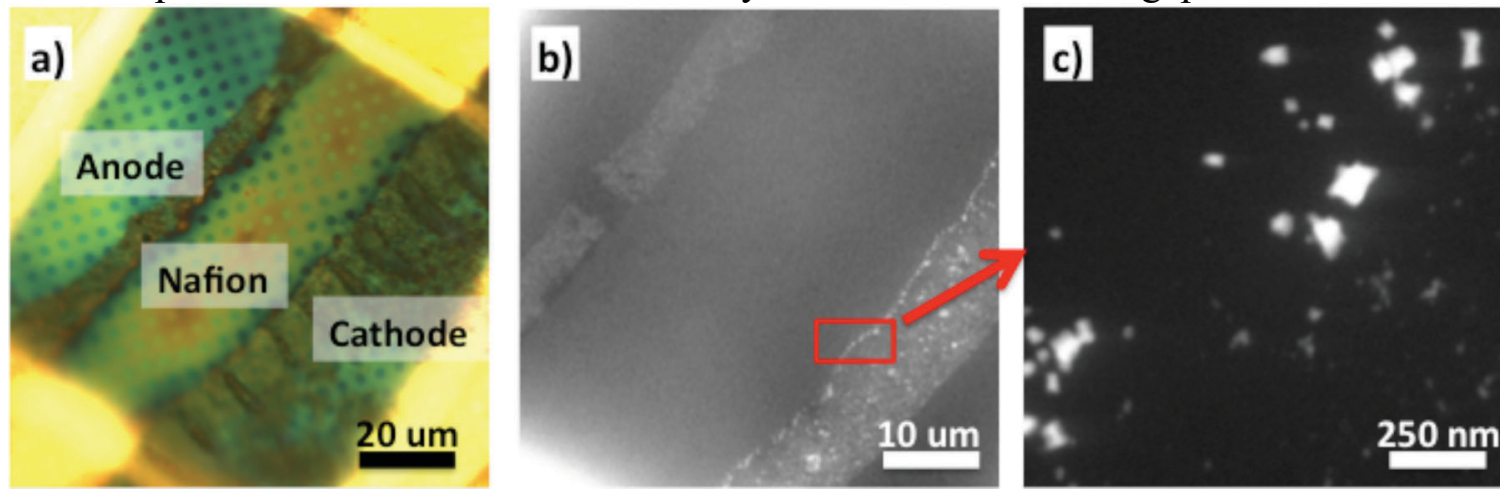

Figure 2. a) Optical image of a cross-sectioned proton-exchange membrane fuel cell. b) AirSEM image of the same fuel cell. c) Higher magnification of the cathode showing the $\mathrm{Pt}_{3} \mathrm{Co}$ catalysts, that have coarsened after 30,000 cycles aging.
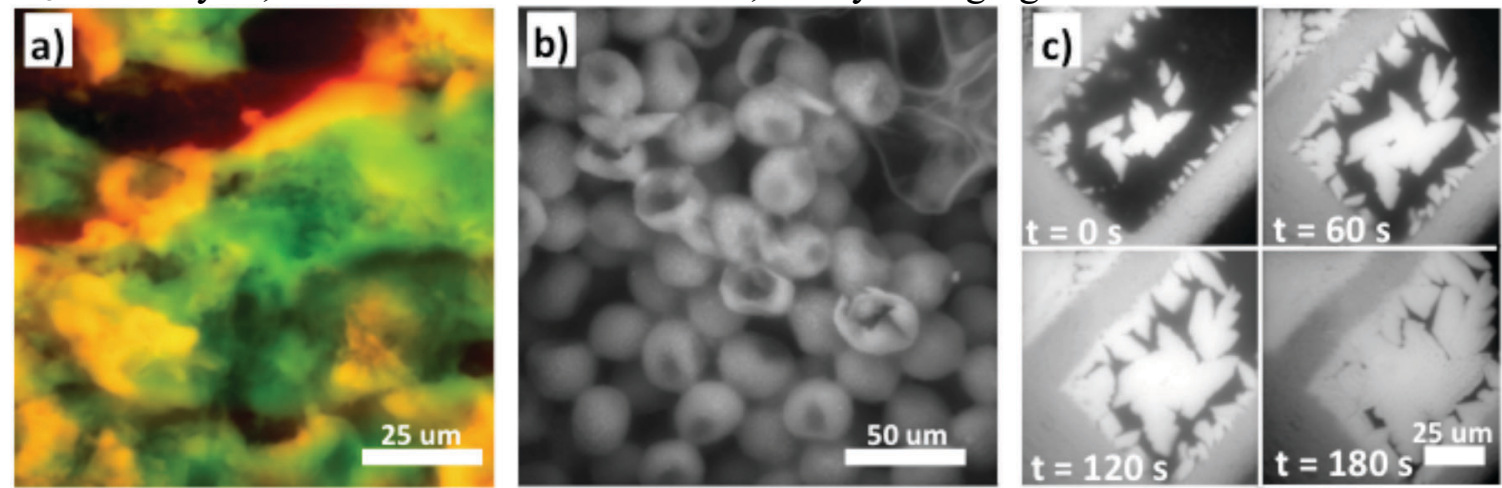

Figure 3. a) Aged Li-ion battery electrode with false color scale overlapping BSE (green) with Surface Detector signals (red) b) Wet moss. c) In-situ growth of $\mathrm{Pb}$ dendrites from solution. 